\title{
VALIDACIÓN DE ESCALA DE VALORACIÓN DEL DOLOR EN RECIÉN NACIDOS EN UNA UNIDAD DE NEONATOLOGÍA
}

\author{
VALIDATION OF A SCALE OF ASSESSMENT OF PAIN \\ IN INFANTS IN NEONATAL UNIT
}

\author{
ANGÉLICA Rivas L.* \\ EDITH Rivas R. ** \\ Luis Bustos M.**
}

\begin{abstract}
RESUMEN
La escala de valoración de dolor de Givens permite determinar presencia y magnitud del dolor que presentan los Recién Nacidos (RN) hospitalizados en Unidades de Cuidados Intensivos (UCI), para así poder valorar de manera constante este signo vital y tomar las medidas necesarias para atenuar la sensación dolorosa. Sentir dolor es un estado no deseable, donde se ve alterado el patrón fisiológico y el estado de conciencia de los niños; se hace necesario aplicar de manera constante escalas que permitan visualizar la magnitud real y tomar medidas de prevención y cuidado enfermero, favoreciendo la tranquilidad de los RN. Esta investigación tuvo como objetivo validar la escala Neonatal Pain Assessment Scale de Givens, en la Unidad de Neonatología, Hospital Dr. Hernán Henríquez Aravena, durante el período abril - septiembre de 2010. El estudio utilizó un diseño de corte transversal, donde se determinó validez y confiabilidad del instrumento. La investigación permitió entregar un instrumento válido y confiable para medir presencia y magnitud del dolor en RN. Así, se determinó un coeficiente alfa de Cronbach de 0,78 y un porcentaje total de varianza de 57,72. El análisis sugirió la eliminación de dos ítemes de valoración, quedando con dos dominios (Conductual y Fisiológico), estableciéndose que la Neonatal Pain Assessment Scale es adecuada para su objetivo de valoración, constituyendo un aporte en unidades de cuidados neonatales, contribuyendo al propósito de mejorar la calidad de hospitalización de estos usuarios y otorgando cumplimiento a la norma ministerial y calidad de los cuidados.
\end{abstract}

Palabras clave: Estudios de validación, evaluación del dolor, recién nacido.

\begin{abstract}
Givens Neonatal Pain Assessment Scale, allows determining the presence and the degree of pain in Newborns (RN) hospitalized in Intensive Care Units (ICU) present, in order to constantly assess the vital signs and take the necessary measures to reduce pain sensation. Feeling pain is an undesirable state, where the physiological pattern and the children consciousness state is altered; it is necessary to consistently apply scales that helps illustrate the real magnitude as well as adopting preventive actions and nursing care, to promote tranquility of the RN. The research, aimed to validate the Givens Neonatal Pain Assessment Scale at Neonatal Unit from Dr. Hernan Henríquez Aravena Hospital, during the period April to September 2010. The study uses a crosssectional design, which determined validity and reliability of the instrument. The research delivers a valid and reliable instrument to measure the presence and degree of pain in neonates. Thus, we determined a Cronbach's alpha of 0,78 and a total percentage of variance of 57,72 . The analysis suggested the elimination of two items of value, leaving with two domains (Behavioral, and Physiological), establishing that the Neonatal Pain Assessment Scale, is suitable for objective assessment, making a contribution in Neonatal Care Units, contributing to the

\footnotetext{
* Enfermera, Hospital Hernán Henríquez A. Temuco. E-mail: ayrivasl@gmail.com

** Enfermera, profesora Departamento de Pediatría y Cirugía Infantil, Facultad de Medicina Universidad de La Frontera, Temuco. Chile.E-mail: erivas@ufro.cl

*** Bioestadístico. Universidad de La Frontera, Temuco. E-mail: lbustos@ufro.cl
} 
purpose of improving the quality of hospitalization of these users and granting Ministerial compliance with the standard and quality of care.

Key words: Validation studies, pain measurement, newborn.

Fecha recepción: 28/03/11 Fecha aceptación: 20/06/12

\section{INTRODUCCIÓN}

La naturaleza del dolor en Recién Nacidos (RN) implica comprender su génesis, considerando que es un estado indeseable, que altera los aspectos fisiológico, psicológico y conductual. En relación a los antecedentes teórico y empíricos, experiencias iniciales se basaron en la concepción de que el RN no sentía dolor o que su sistema nervioso aferente era inmaduro, existiendo un olvido de la sensación dolorosa. Actualmente, hay evidencia que las dolencias crónicas generan severo impacto psicológico, emocional - afectivo, personal y familiar (1). El Ministerio de Salud ha catalogado la valoración del dolor como el quinto signo vital (2), lo que hace aún más importante su valoración. Lo anteriormente expuesto induce a reflexionar, reforzando la idea de que el profesional sanitario debe tratar con consideración y respeto a sus pacientes (3), practicando la Ética de Mínimos, enfocándose en la aplicación de instrumentos confiables y validados, que permitan descubrir presencia y nivel de dolor en los RN sometidos a procedimientos realizados durante su hospitalización, debido a que el ambiente hospitalario concede una situación especial, donde el dolor experimentado es potenciado por la ansiedad, temor, tristeza y molestia del mismo usuario o de los padres de éste (4). Por otra parte, la exposición a un medio hostil y ruidoso, como es el caso de una UCI, dificulta la organización del cerebro en desarrollo. Además, los padres se someten a tal impacto emocional, lo cual modifica el proceso de crianza (5). Por otra parte, es conocido que en el RN hospitalizado, la ausencia de percepción de los ciclos día
- noche acentúa la ansiedad (4), además de alterar la fisiología del sueño - vigilia, modificando niveles bioquímicos de suma importancia, alterando el normal proceso de crecimiento y desarrollo descritos anteriormente.

Debido a lo expuesto anteriormente, resulta importante validar la escala Neonatal Pain Assessment Scale de Givens S (6), en una Unidad de Cuidado Intensivo Neonatal.

En este contexto, es relevante mencionar que la medición en salud es habitual en la praxis diaria, recordando que como hito psicosocial requiere agudizar sus instrumentos para obtener, de forma válida y confiable, la subjetividad de los usuarios (7).

\section{Objetivo general:}

Validar la Neonatal Pain Assessment Scale de Givens en Recién Nacidos de una Unidad de Cuidado Intensivo Neonatal.

Objetivos específicos:

- Describir los ítemes de la Neonatal Pain Assessment Scale de Givens

- Adaptar cultural e idiomáticamente la escala

- Caracterizar el perfil de la muestra y

- Determinar propiedades psicométricas (validez y confiabilidad) de la Neonatal Pain Assessment Scale de Givens.

\section{MATERIAL Y MÉTODO}

Estudio de corte transversal, donde se determinó validez y confiabilidad del instrumento "Neonatal Pain Assessment Scale de 
Givens" (6), en una muestra probabilística, no intencionada, de 112 valoraciones de dolor en RN, hospitalizados en UCI Neonatal del Dr. Hernán Henríquez Aravena, durante el período abril - septiembre de 2010 y que cumplíeron con criterios éticos y clínicos. Las valoraciones fueron realizadas por la investigadora en conjunto con enfermeras con al menos dos años de experiencia en UCI.

Recolección de datos a través de la Neonatal Pain Assessment Scale de Givens (6), la cual presenta formato tipo Likert, con rango de $0-20$ puntos, valor menor a 4: sin dolor; 5 - 8: dolor moderado y mayor o igual a 9: dolor intenso (8). Constructo o parámetro conductual compuesto por 6 ítemes: duerme durante la hora precedente a la valoración, expresión facial de dolor, actividad motora espontánea, tono global, consuelo y llanto. Fisiológico compuesto por 4 ítemes: frecuencia cardíaca, presión arterial sistólica, frecuencia respiratoria y cualidades y saturación de oxígeno. Adicionalmente, se consignaron aspectos sociodemográficos: edad cronológica y gestacional, sexo, peso de nacimiento; presencia de analgesia y elementos invasivos.

Los datos fueron ingresados en planilla Excel. Análisis estadístico, a través de SPSS para Windows $15.0 \mathrm{v}$, extrayéndose frecuencias y porcentajes de los aspectos demográficos y variables o parámetros conductuales y fisiológicos de la escala.

Para validación de la escala, se utilizó retrotraducción del instrumento (inglés-español-inglés-español), validez de fachada (a través de pauta chequeo, por 6 expertos en el área) y análisis factorial exploratorio a través de la técnica de rotación Varimax (de varian- za máxima) y normalización con Kaiser (9).

Para determinar la confiabilidad se utilizó el coeficiente alfa de Cronbach, considerando al instrumento en su conjunto y para los dominios obtenidos del análisis factorial (10).

El estudio contó con autorización del Comité de ética del Servicio Salud y del establecimiento hospitalario. Además, consideró consentimiento informado, resguardando la confidencialidad y el anonimato. Se veló por el respeto a los principios de no-maleficencia y beneficencia, no existiendo riesgo para los RN (11).

\section{RESULTADOS}

A través del método de traducción y retrotraducción del instrumento $(12,13)$, se obtuvo la escala para el estudio, estableciéndose la equivalencia con la versión original.

Respecto a la validación de fachada: en un primer momento los expertos consultados (psicólogos, enfermeras y metodólogo) concordaron en el $80 \%$ de los aspectos valorados en la pauta chequeo, modificándose en los aspectos de redacción y explicación de los ítemes. En la reevaluación mostraron concordancia cercana al 100\%.

En relación al perfil de la muestra, el $61,6 \%$ de RN correspondían a hombres. Un $98,2 \%$ se encontraba con elementos invasivos y $8 \%$ presentó analgesia al momento de aplicar la escala. Un 35,7\% estaba conectado a sistema de ventilación mecánica, un $8 \%$ de los RN en estado postoperatorio (Tabla 1). En edad gestacional, se obtuvo un promedio

Tabla 1. Perfil clínico de hospitalización de 112 RN. Unidad de Cuidados Neonales Hospital Dr. H.H.A. Temuco, 2010.

\begin{tabular}{lrr}
\hline Variables & f & \% \\
\hline Mujer & 43 & 38,4 \\
Presentan elementos invasivos & 110 & 98,2 \\
Presentan analgesia & 9 & 8,0 \\
Presentan ventilación mecánica & 40 & 35,7 \\
Postoperados & 9 & 8,0 \\
\hline
\end{tabular}


de 31,6 semanas de gestación con una DS de 4,6 semanas (mínimo de 24 semanas y máximo de 40). La edad cronológica fue de 21,9 días con una DS de 30,7 (mínimo 1 y máxi- mo 211 días). El peso promedio de los $\mathrm{RN}$, fue de 1.645,5 gramos con una DS de 876,7, adecuado para la edad gestacional promedio (Tabla 2).

Tabla 2. Perfil sociodeográfico de RN hospitalizados en Unidad de Cuidados Neonales Hospital Dr. H.H.A. Temuco, 2010.

\begin{tabular}{lccc}
\hline Variables & Media \pm DS & Mínimo & Máximo \\
\hline Edad gestacional (semanas) & $31,6 \pm 4,6$ & 24 & 40 \\
Edad cronológica (días) & $21,9 \pm 30,7$ & 1 & 211 \\
Peso de nacimiento (gramos) & $1.645,5 \pm 876,7$ & 680 & 3.700 \\
Ptje. escala valoración & $5.47 \pm 3.55$ & 0 & 16 \\
\hline
\end{tabular}

La puntuación promedio en la escala de Givens fue de 5,5 puntos, con un rango de 0 a 16 , no encontrándose $\mathrm{RN}$ con puntaje máximo de la escala.

Respecto al constructo o parámetros conductual valorado, se encontró un $13,4 \%$ de $\mathrm{RN}$ con gran alteración del sueño, $8 \%$ con expresión marcada de dolor y no hubo RN con llanto vigoroso. Los aspectos conduc- tuales actividad motora espontánea, duerme durante la hora precedente y tono global fluctuaron entre un $10,7 \%$ y $14,3 \%$. En el constructo fisiológico, los porcentajes más alterados fueron saturación de oxígeno y frecuencia respiratoria con 29,5 y $21,4 \%$, respectivamente. Frecuencia cardíaca 6,2\% y presión arterial media 2,7\%. (Tabla 3).

En relación al puntaje total obtenido en

Tabla 3. Constructos de la escala de evaluación del dolor neonatal de Givens S., según nivel de respuesta. Neonatología H. Dr. H.H.A. Temuco, 2010.

\begin{tabular}{lrrrrrr}
\hline Constructos\Puntaje & \multicolumn{3}{c}{$\mathbf{0}$} & $\mathbf{1}$ & $\mathbf{2}$ \\
\cline { 2 - 7 } & $\mathbf{f}$ & $\mathbf{0}$ & $\mathbf{f}$ & $\mathbf{0}$ & $\mathbf{f}$ & $\mathbf{\%}$ \\
\hline Conductuales & & & & & & \\
Duerme durante la hora precedente & 68 & 60,7 & 29 & 25,9 & 15 & 13,4 \\
Expresión facial de dolor & 52 & 46,5 & 51 & 45,5 & 9 & 8,0 \\
Actividad motora espontánea & 41 & 36,6 & 59 & 52,7 & 12 & 10,7 \\
Tono global & 53 & 47,3 & 43 & 38,4 & 16 & 14,3 \\
Consuelo & 32 & 28,6 & 70 & 62,5 & 10 & 8,9 \\
Llanto & 91 & 81,3 & 21 & 18,7 & 0 & 0 \\
Fisiológicos & & & & & & \\
Frec. cardiaca & 70 & 62,5 & 35 & 31,3 & 7 & 6,2 \\
Presión arterial media & 106 & 94,6 & 3 & 2,7 & 3 & 2,7 \\
Frec. respiratoria & 50 & 44,7 & 38 & 33,9 & 24 & 21,4 \\
Saturación de $\mathrm{O}_{2}$ & 73 & 65,2 & 6 & 5,3 & 33 & 29,5 \\
\hline
\end{tabular}

la escala de Givens, se observó que un 44,6\% de los RN no presentó dolor, un 40,2\% dolor moderado y $15,2 \%$ dolor intenso.
En relación a determinación de validez y confiabilidad, inicialmente, se estudió la escala original con los 10 ítemes que la confor- 
man. Para el análisis factorial exploratorio, la determinante de esta matriz fue mayor a cero y el valor del estadístico Kaiser-Meyer-Olkin (KMO) fue 0,8 (muy bueno). Por tanto, se esperaría un buen ajuste factorial, el cual arrojó tres componentes, cuyo porcentaje de varianza acumulada fue de 59\%. Los ítems de los factores encontrados fueron: Factor 1: Ítemes 5, 6, 10, 9, 1; Factor 2: Ítemes 3, 4, 2, 7 y Factor 3: ítem 8. Al realizar análisis de consistencia interna con el alfa de Cronbach, se encontró que al eliminar el ítem 7 , aumenta la confiabilidad de 0,73 a 0,76 . En un segundo análisis, se observó que al eliminar el ítem 8 , la confiabilidad de la escala ascendía a 0,78.

De esta forma, con los ocho ítemes que se seleccionan, los resultados del nuevo análisis factorial fueron los siguientes: Los dos dominios finales de la escala presentaron porcentajes de varianza similares. Ambos conforman el 57,7\% de varianza acumulada (Tabla 3). Los componentes rotados de cada uno de ellos se explican en la Tabla 4. El factor 1, conformado por las preguntas 6, 5, 10 y 9 , cuyo porcentaje de varianza fue 28,9 . El factor 2, constituido por las preguntas 3, 4, 2 y 1 , con porcentaje de varianza de 28,8 (Tabla 4), desapareciendo el factor 3. A través del análisis de consistencia interna de la escala, se determinó un alfa de Cronbach de 0,78. El factor 1 presentó un alfa de 0,62 y 0,79 para el factor 2 (Tabla 5).

Tabla 4. Análisis factorial exploratorio de la escala de evaluación del dolor neonatal de Givens S. Neonatología H. Dr. H.H.A. Temuco, 2010.

\begin{tabular}{lc}
\hline Componente & \% de la varianza \\
\hline 1 & 28,9 \\
2 & 28,8 \\
\hline
\end{tabular}

Tabla 5. Matriz de componentes rotados de la escala de evaluación del dolor neonatal de Givens S. Neonatología H. Dr. H.H.A. Temuco, 2010.

\begin{tabular}{lcc}
\hline & \multicolumn{2}{c}{ Componentes } \\
Ítemes & $\mathbf{1}$ & $\mathbf{2}$ \\
\hline Llanto & $\mathbf{0 , 7 6}$ & 0,01 \\
Consuelo & $\mathbf{0 , 7 4}$ & 0,01 \\
Saturación de $\mathrm{O}_{2}$ & $\mathbf{0 , 6 0}$ & 0,25 \\
Frecuencia respiratoria & $\mathbf{0 , 5 4}$ & 0,29 \\
Actividad motora espontánea & 0,13 & $\mathbf{0 , 8 6}$ \\
Tono global & $-0,01$ & $\mathbf{0 , 8 3}$ \\
Expresión facial de dolor & 0,51 & $\mathbf{0 , 6 7}$ \\
Duerme durante la hora precedente & 0,50 & $\mathbf{0 , 5 2}$ \\
\hline Método de extracción: análisis de componentes principales. \\
Método de rotación: rotación de Varimax con Kaiser.
\end{tabular}

Tabla 6. Distribución de resultados de los dominios de la escala de evaluación del dolor neonatal de Givens S. Neonatología H. Dr. H.H.A. 2010.

\begin{tabular}{lccccc}
\hline & Dominios & $\mathbf{N}^{\circ}$ de ítemes & Ítemes & \% Varianza & Consistencia interna \\
\hline Escala de dolor de Givens S. & Total & 8 & $1-8$ & 57,7 & 0,78 \\
& 1 & 4 & $6,5,10,9$ & 28,9 & 0,62 \\
& 2 & 4 & $3,4,2,1$ & 28,8 & 0,79 \\
\hline
\end{tabular}




\section{DISCUSIÓN Y CONCLUSIÓN}

La experiencia de dolor en recién nacidos, situación indeseable, presenta dificultad en su valoración, la que resulta compleja por su carácter subjetivo. Se han realizado esfuerzos en la creación de escalas que lo puedan medir de manera confiable, permitiendo que se puedan tomar las estrategias para compensar la dolencia, constituyendo un aporte en las Unidades de Cuidado.

La validación de la Escala de Valoración de Dolor Neonatal de Givens (Neonatal Pain Assessment Scale) ha evidenciado resultados de una realidad local. Al aplicar las pruebas de validez y confiabilidad a la escala en estudio, éstas arrojaron resultados satisfactorios y positivos, fundamentados en un porcentaje de varianza acumulada y consistencia interna, con valores de $57,7 \%$ y 0,78 , respectivamente. Asimismo, dentro de los resultados obtenidos, el análisis sugirió la eliminación de dos ítemes de la escala original, 7 y 8 , entendiéndose como no relevantes para el objetivo fisiológico que se quiere medir.

Respecto de la presencia y nivel de dolor, en este estudio se encontró un $15,2 \%$ de dolor intenso y un 40,2\% de nivel moderado. En contraste con el estudio realizado por Villamil et al. en Colombia, quienes encontraron en una Unidad Neonatal local un 3,4\% de $\mathrm{RN}$ con dolor moderado y un $95,7 \%$ con dolor intenso (8), visualizándose la diferencia significativa en los porcentajes al utilizar igual instrumento, donde se debe tener en cuenta que la realidad de los Centros Hospitalarios Neonatales son diferentes. Por otra parte, en un estudio realizado por Ibarra et al., en España, se evidenció que al aplicar la escala de Givens (6), los constructos conductuales poseían relevancia en las evaluaciones, incluso más que los fisiológicos (14), lo que avala el porcentaje de validez de este dominio de ítemes y su confiabilidad, resultando de importancia el aplicarla en diferentes circunstancias y usuarios, proponiendo la reali- zación de valoraciones multicéntricas.

Cada Unidad de Cuidado Neonatal utilizará el instrumento que considere simple y sencillo de aplicar por su personal (15), desarrollando estrategias que colaboren en la disminución de presencia y magnitud del dolor en los neonatos, de manera natural, sin esperar que sea determinado un mandato ministerial, en circunstancias que debiera ser efectuado a priori, como manifestación de cuidados humanizados. Un profesional sanitario debe buscar estrategias y conductas con gestión de prevención o minimización de la sensación dolorosa, tales como evitar procedimientos innecesarios, utilizar soluciones orales dulces, masajes relajantes, así como amamantar al pecho de su madre mientras se realiza una acción dolorosa.

Finalmente, se espera que a través de esta investigación, se fomente la motivación por indagar en aspectos propios de Enfermería que constituyen parte del accionar, donde exista plena seguridad que la acción basada en la evidencia empírica, científica, es el camino a lograr la impecabilidad del ejercicio de la profesión. Asimismo, este estudio patenta una línea de acción en el cuidado y en la construcción del saber enfermero.

\section{REFERENCIAS}

1. Lima G, Aldana L, Casanova P, Casanova $P$, Casanova C. Inducción y medición del dolor experimental. Rev. Cub. Med. Mil. [revista en internet]. 2003 [citado 17 mayo 2010]; 32 (1). Disponible en: http://scielo.sld.cu

2. Ministerio de Salud de Chile. MINSAL. Circular Ministerial 2a/46: Evaluación del Dolor como Quinto Signo Vital. 2004.

3. Grzona M. Prematuros Extremos: ¿Es posible establecer un límite de viabilidad? Acta bioeth [revista en internet]. 2006 [citado 2 enero 2010]; 12 (1): 101- 
111. Disponible en:_http://www.scielo.cl

4. Tutaya A. Dolor en Pediatría. Pediátrica [revista en internet]. 2002 [citado 15 enero 2010]; 4 (2): 27-40. Disponible en: http://sisbib.unmsm.edu.pe/BvRevistas/ Paediatrica/v04_n2/dolor.ht

5. Perapoch L, Pallás A, Sillo M, Moral M, Pumarega F, Castro B et al. Cuidados centrados en el desarrollo. Situación en las unidades de neonatología de España. An Pediatr (Barc). 2006; 64 (2): 132-9.

6. Givens Bell S. Neonatal Pain Assessment Scale. 1993-1994. [Internet]. [citado 15 enero 2010]. Disponible en: http://www. vachss.com/guest_dispatches/neonatal_ pain_tables.html

7. Guerrero L. De un enfoque holístico a la cuestión de la neuroplasticidad. ARS méd. (Santiago). [revista en internet]. 2000 [citado 30 diciembre 2010];3(3):6671. Disponible en: http://escuela.med. puc.cl/publ/arsmedica/arsmedica.html

8. Villamil A, Ríos M, Bello S, López NC, Pabón IC. Valoración del dolor neonatal: una experiencia clínica. Aquichán. 2007; 7(2): 120-129.

9. Vergara M. Elaboración y validación de un cuestionario de calidad de vida para los familiares que viven con pacientes con enfermedad inflamatoria intestinal. [Tesis Magíster]. [Barcelona]: Universidad Autónoma de Barcelona;1999. p. 31.

10. Piña J, Rivera B. Validación del cuestio- nario de apoyo social funcional en personas seropositivas al VIH del noroeste de México. Cienc. enferm. 2007; XIII (2): 53-63.

11. González M. Aspectos éticos de la investigación cualitativa. Rev. Iberoam. Educ. [revista en internet]. 2002 mayo-agosto [citado 30 diciembre 2010]; 29: 85-103. Disponible en: http://www.rieoei.org/ rie29a04.PDF

12. Guillemin F, Bombardier C, Beaton D. Crosscultural adaptation of health-related quality of life measures: literature review and proposed guidelines. J Clin Epidemiol. 1993; 46 (12): 1417-1432.

13. Brislin W. The wording and translation of research instruments. En Lonner W, Berry W, eds. Field methods in crosscultural research. Beverly Hills, CA: Sage publications; 1986; p. 137-164.

14. Ibarra F, Antinio M, Llanos I, Quesada C, Martínez F, Bonillo F. Escala de valoración del dolor en neonatología Tempus Vitalis. Revista Internacional para el Cuidado del Paciente Crítico. 2004; 4 (1).

15. Flores MA. Neurofisiología del Dolor en el feto y en el recién nacido. Foro de Investigación y Tratamiento del Dolor para la Comunidad Médica. [Internet] [citado 27 abril 2010]. Disponible en: http://www.intramed.net 